\title{
A review of planar scissor structural mechanisms: geometric principles and design methods
}

\author{
Feray Maden ${ }^{1 *}$, Koray Korkmaz ${ }^{1}$ and Yenal Akgün² \\ ${ }^{1}$ Department of Architecture, Izmir Institute of Technology, Gülbahçe Kampüsü, 35430 Izmir, Turkey \\ ${ }^{2}$ Department of Interior Architecture, Gediz University, Seyrek Kampusu Menemen, 35665 Izmir, Turkey
}

This study deals with a review of planar scissor structural mechanisms (SSMs) and reports on how they can be easily transformed from a stowed to a deployed configuration. These mechanisms have an important transformation capacity of their extension and rotation properties, and many examples have been proposed that vary in size, type and geometry. Although there are many studies dealing with designing new planar or spatial SSMs and their calculation methods, there is no systematic study demonstrating the basic typologies, geometric principles, design rules and constraints of such SSMs. Further, current calculation methods are based on the inductive approach in which the dimension of one scissor unit (SU) is given, but the span of the whole structure is found later according to the number of SUs that are used to assemble the structure. However, this approach is not convenient for architectural applications, because it requires a deductive approach in which the dimensions and required number of SUs are calculated according to defined span length. On the basis of this concept, this article, first, analyses the geometric design of SSMs systematically in terms of their possible configurations and then develops trigonometric calculation methods for different types of SSMs, using a deductive approach.

Keywords: Deployable structures; geometric principles of scissor structures; planar scissor structural mechanisms

\section{INTRODUCTION}

A planar scissor structural mechanism (SSM) is formed by scissor units (SUs). Each SU consists of two straight bars that are connected to each other at their intermediate points by a revolute joint. Called a scissor hinge, this revolute joint allows large geometric transformations. By altering the location of the scissor hinge, three distinct basic unit types are obtained: translational, polar and angulated units (Figure 1). For translational units, unit lines that connect the upper and lower nodes of one SU must be parallel and remain so during deployment. On the other hand, for polar and angulated units, they intersect at a single point. The difference between these two unit types is that angulated units consist of two identical angulated bars, whereas polar units consist of straight ones.

The concept of scissor structures was first introduced in 1961 by Spanish architect Pinero. Using simple SUs, Pinero designed various reticulated planar and spatial grids as mobile theatres, pavilions and exhibition buildings (Pinero, 1961). Pinero's structures have needed additional cables to be stabilized at their final configurations. In
1974, Zeigler solved this problem and proposed a selfsupported dome-shaped scissor structure that does not require additional members for stabilization. Pinero's and Zeigler's ideas have lead other researchers to investigate similar structures. For instance, Clarke (1984) developed a novel spatial unit called the 'Trissor', by intersecting three SUs; he designed a hemispherical dome, using this new unit. Escrig and Valcarcel developed new spherical grid structures composed of two-way and three-way scissors and proposed several connection details for these designs (Escrig, 1984, 1985; Escrig and Valcarcel, 1986b, 1987). Following the studies of Escrig, Chen et al. (2002) and Gutierrez and Valcarcel (2002) developed two-way spatial scissor systems in order to obtain planar or dome-like scissor shells. Beside the employment of scissor systems as portable roof structures, Kwan (1995) and Kwan et al. (1993) used scissor systems in space technologies and developed satellite panels with planar polar scissors. He used additional cable elements to move and fix the system.

Hoberman (1990) made a considerable advance in the design of scissor structures when he developed the angulated element. While it is not possible to design radially deploying 


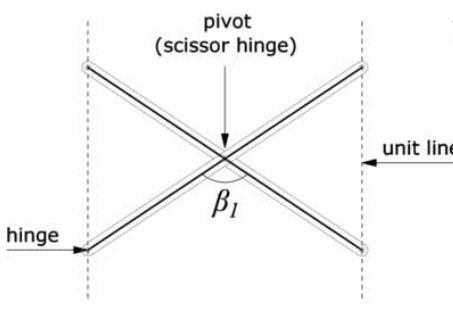

( $\beta_{1}, \beta_{2}$ and $\beta_{3}$ are deployment angles)

Translational Unit

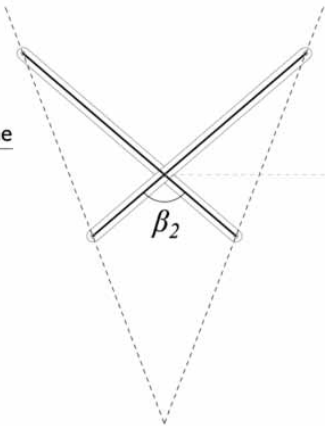

Polar Unit

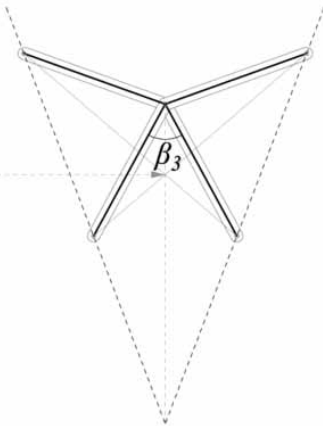

Angulated Unit

Figure 1|Basic scissor units (SUs)

closed-loop structures with translational or polar units because of their deployment behaviours, it can be designed with angulated units (Buhl et al., 2004). Hoberman designed the Iris Dome and Hoberman Sphere, using the angulated element. Hoberman's pioneering idea of the angulated element lead You and Pellegrino (1997) to make further progress on scissor structures. They investigated the multiangulated element whose elements have more than one kink angle. Gan and Pellegrino (2003, 2006) improved the design of Hoberman, explained the geometry of structural mechanisms in analytical and numerical ways and proposed several novel concepts.

In addition to these key studies, there are also some other innovative works on scissor structures: For instance, Kokawa and Hokkaido (1997) designed a convertible scissor structure called Cable Scissors Arch (CSA), which consists of three-hinged arch scissors and zigzag flexible cables with pulleys installed at connection points between the SUs. By winding up the cable, using a winch, CSA can lift up and fall down. Van Mele et al. (2007) have combined scissor structures with membrane structures and designed novel canopy and covering systems. Atake (2000) has connected SUs spatially and developed a novel spatial scissor system. This novel system can constitute scissor shells as well as polyhedral geometries. Because of its spatial geometry, this system is very convenient for use as a structural element. Akgün et al. (2010a) developed modified scissorlike element (M-SLE) and designed a transformable planar scissor roof structure. This adaptive structure can form various shapes without changing the size of the covered area. They adapted the principles of M-SLE to spatial polar scissor systems and developed a scissor shell that can transform from various double curved and arch-like shapes (Akgün et al., 2010b). In addition to these studies, polygonal hyperboloids of Al Khayer and Lalvani (1998), Florin System of Dieguez and Cozar (1998) and the master thesis of Block (2003), which integrates scissor systems, pneumatic muscles and membrane systems, are other remarkable examples of innovative designs of deployable scissor structures.
Besides research on the development of new SUs and mechanisms, some studies have focused on explaining the geometric, structural and kinematic behaviours of the current examples. As pioneers in this area, Escrig and Valcarcel (1987, 1993) derived foldability conditions for scissor structures and developed matrix calculation programs to analyse the stress and movements in the deployed configuration (Escrig and Valcarcel, 1986a). Using purely geometric approaches, Zanardo (1986) and Langbecker (1999) investigated the planar scissor systems and extended the foldability condition of Escrig to determine the foldability of translational, cylindrical and spherical configurations and to analyse their kinematics. Furthermore, Langbecker designed several models of positive and negative curvature structures, using compatible translational units. Krishnapillai generalized the concept of Zeigler and found a number of configurations satisfying the requirement of zero-stress in folded and deployed configurations (Gantes, 2001). Further, Gantes and his colleagues investigated scissor structures systematically and developed quantitative design and geometric principles for both planar and spatial scissor structures (Gantes et al., 1993, 1994; Gantes and Konitopoulou, 2004). Patel and Ananthasuresh (2007) have explained the geometry and kinematics of scissor systems based on angulated elements and plates. Finally, Kaveh and Davaran (1996), Nagaraj et al. (2009) and Zhao and Feng (2009) made a deep geometric and kinematic analysis of planar and spatial SUs and simple grids, using matrix-based methods. In fact, many calculation methods for designing both planar and spatial SSMs have been developed using simple geometric approaches based on trigonometric, analytic or matrix methods. Rather than reviewing these solutions one by one, this article seeks to present a basic geometric design methodology using a basic trigonometry that may be easily adapted to architectural applications of such systems.

The review discussed above shows that many types of SSMs have been discovered. Each type has different deployment configurations. Such configurations are directly related to the type of SUs that are used to assemble the structure. For 
instance, rectilinear SSMs are obtained using translational units whereas curvilinear ones are generated using polar units. On the other hand, using angulated units, both rectilinear and curvilinear SSMs can be obtained. To understand geometric design principles of such SSMs, it is necessary to examine general deployability conditions of scissor structures and analyse basic unit types of translational, polar and angulated in detail.

\section{GENERAL DEPLOYABILITY CONDITION}

The crucial part of the overall design process of deployable scissor structures is its geometric design, because, it not only consists of choosing a desired geometric shape, but also includes the selection of the type of basic unit. There are some geometric conditions (deployability constraints) for foldability of planar SSMs using translational or polar units. One of these requirements of scissor structures is that the configuration is capable of being stored in a compact shape. As shown in Figure 2, SUs will have one dimension in the compact shape; therefore, $B_{0}, C_{0}, A_{1}, B_{1}$, $C_{1}, A_{2}, B_{2}, C_{2}, A_{3}, B_{3}$ and $C_{3}$ will be collinear. The distance between $C_{1} B_{1}$ can be found using the cosine rule

$$
\begin{aligned}
a_{i}^{2}+b_{i}^{2}-2 a_{i} b_{i} \cos \left(\theta_{i}\right)= & a_{i+1}^{2}+b_{i+1}^{2} \\
& -2 a_{i+1} b_{i+1} \cos \left(\theta_{i+1}\right)
\end{aligned}
$$

In the compact shape $\theta_{i}=\theta_{i+1}=\pi$, thus, the above equation is reduced to

$$
a_{i}+b_{i}=a_{i+1}+b_{i+1}
$$

Derived by Escrig, this equation states that the sum of the lengths of bars on both sides of the unit line should be equal. However, Escrig's equation can be applied to units composed of straight bars, and not to those of angulated bars. Hence, it uses purely a geometric approach and does not guarantee that stresses will be kept to an acceptable level during the deployment process under the condition that the materials and the sizes of members' change (Rosenfeld and Logcher, 1988).

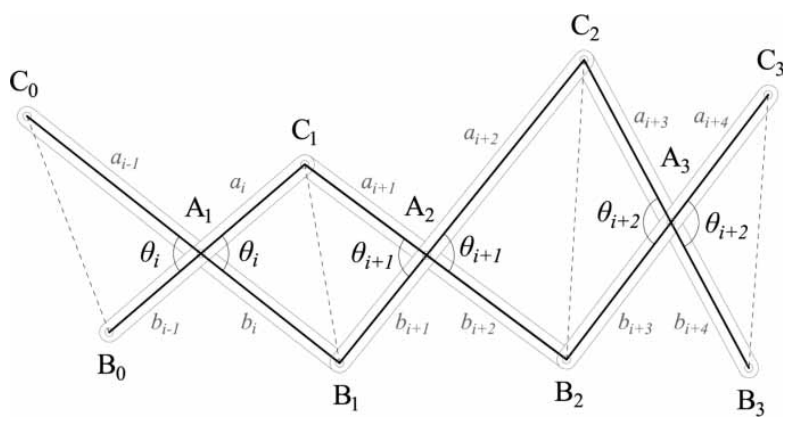

Figure 2 | Deployability condition for SUs

\section{SU TYPES}

\section{Translational units}

Consisting of SUs with straight bars, rectilinear SSMs can only translate without any rotation. The main rule to meet this condition is that all unit lines must be parallel to each other during and after the deployment process. According to bar lengths and the location of scissor hinge, there can be various types of rectilinear SSMs. Some of these types are investigated in this study.

The first type of rectilinear SSM consists of SUs whose bars are identical and whose scissor hinge is located at the midpoints of the bars (Figure 3). By interconnecting such SUs, a rectilinear SSM that constitutes a perfect planar surface is generated. This SSM is called lazy tong (De Temmerman, 2007). Its condition can be formulized as

$$
\begin{aligned}
a_{i-1} & =b_{i-1}=a_{i}=b_{i}=a_{i+1} \\
& =b_{i+1}=\cdots=a_{n}=b_{n}=l
\end{aligned}
$$

To solve the system, at least three of these variables should be determined: span of the whole system $(S)$, span of one $\mathrm{SU}(s)$, number of $\mathrm{SU}(N)$, angles between bars $(\theta)$, deployment angle $(\beta)$, unit thickness $(t)$ and the length of bars $(L)$. For the structure in Figure 3, the known parameters are $L(2 l), S$ and $\theta$. It should be noted that angles $\theta$ and $\beta$ are same for each SU. $s$ can be found using the formula

$$
s=2 l \cos \frac{\theta}{2}
$$

After finding $s, N$ can be easily calculated. Further, $\beta$ can be derived according to the cosine rule of triangle $A_{1} B_{0} B_{1}$,

$$
N=\frac{S}{s}
$$

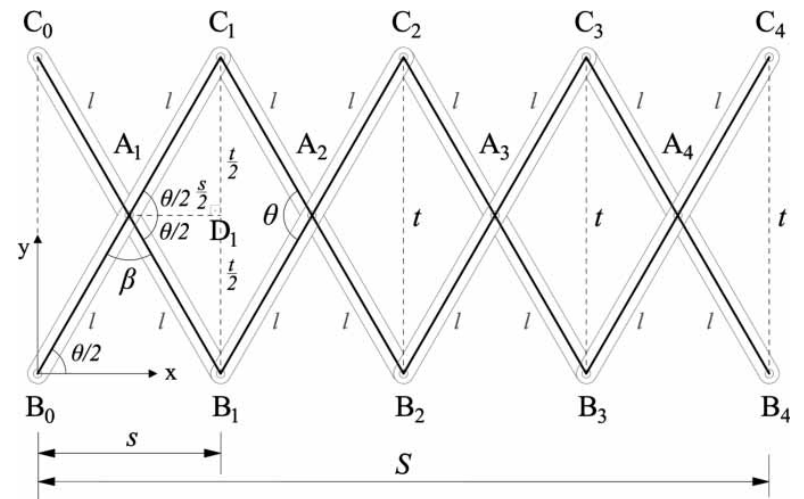

Figure 3|Rectilinear SSM with identical bars and with scissor hinges at their midpoints 


$$
\beta=\cos ^{-1}\left[1-\frac{s^{2}}{2 l^{2}}\right]
$$

The unit thickness $t$ can be found using Pythagoras' theorem of triangle $C_{1} A_{1} D_{1}$. Further, coordinates of the $N$ th $\mathrm{SU}$ are calculated according to origin $B_{0}$ of the system:

$$
\begin{gathered}
t=2 \sqrt{l^{2}-\frac{s^{2}}{4}} \\
x_{B_{N}}=x_{C_{N}}=N 2 l \cos \frac{\theta}{2} \\
y_{B_{N}}=0 \quad \text { and } \quad y_{C_{N}}=2 l \sin \frac{\theta}{2}
\end{gathered}
$$

The second type of rectilinear SSM consists of SUs whose bar lengths are different, but again the scissor hinge is at the midpoints of the bars. In this type, the system still translates and unit lines of hinges remain parallel during the deployment process (Figure 4). The conditions for this type can be written as

$$
\begin{aligned}
& a_{i-1}=b_{i}=a_{i+1}=b_{i+2}, \ldots, a_{n}=b_{n+1}=l_{1} \\
& b_{i-1}=a_{i}=b_{i+1}=a_{i+2}, \ldots, b_{n}=a_{n+1}=l_{2}
\end{aligned}
$$

The known parameters for Figure 4 are $S, L_{1}\left(2 l_{1}\right), L_{2}\left(2 l_{2}\right)$ and $\theta\left(\theta_{1}+\theta_{2}\right)$. To solve the system, unknown parameters of $s, t, N$ and $\beta$ of SUs should be found according to the reference system whose origin is at point $B_{0}$ :

$$
s=2 l_{2} \cos \theta_{1}
$$
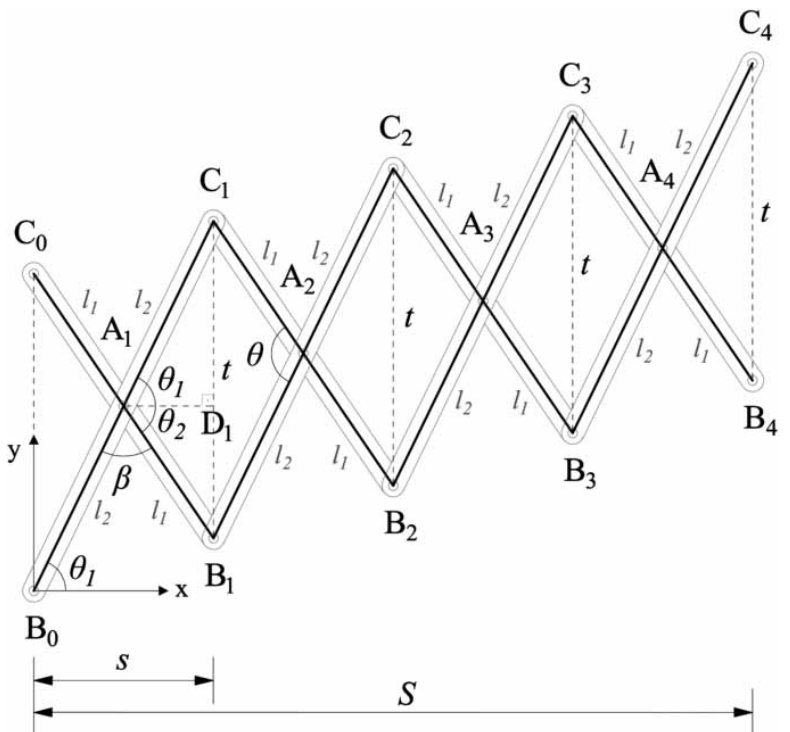

Figure 4 | Rectilinear SSM with different bars and with scissor hinges at their midpoints

$$
\begin{gathered}
N=\frac{S}{S} \\
t=\sqrt{l_{1}^{2}+l_{2}^{2}-2 l_{1} l_{2} \cos \theta} \\
\beta=\pi-\theta
\end{gathered}
$$

When $N$ numbers of SUs are connected to the system, the coordinates on the $N$ th point are:

$$
\begin{gathered}
x_{B_{N}}=x_{C_{N}}=N\left(2 l_{2} \cos \theta_{1}\right) \\
y_{B_{N}}=N\left(\left(2 l_{2} \sin \theta_{1}\right)-t\right) \\
y_{C_{N}}=t+N\left(\left(2 l_{2} \sin \theta_{1}\right)-t\right)
\end{gathered}
$$

By combining the geometric principles of the first and second types of rectilinear SSMs, the third type is obtained (Figure 5). In this type, bar lengths of one SU are different and the scissor hinge is at the midpoints of the bars, as in the second type. The system translates as it does in the first type, but the construction method is completely different from that of the first two types, because the basic SU is not repeated through the SSM. The latter unit is connected to the previous one by reversing the last unit. Because all unit lines are parallel to each other and remain so during the deployment, the system forms a planar surface. Its condition is that

$$
\begin{aligned}
a_{i-1} & =b_{i}=a_{i+2}=b_{i+1}=a_{i+3} \\
& =b_{i+4}=a_{i+6}=b_{i+5}=l_{1} \\
b_{i-1} & =a_{i}=b_{i+2}=a_{i+1}=b_{i+3} \\
& =a_{i+4}=b_{i+6}=a_{i+5}=l_{2}
\end{aligned}
$$

The given parameters of SSM in Figure 5 are $S, L_{1}\left(2 l_{1}\right)$, $L_{2}\left(2 l_{2}\right)$ and $\theta\left(\theta_{1}+\theta_{2}\right)$. Because the construction parameters of this SSM are same as those of the second type of rectilinear SSM, the unknowns of system $s, N, t$ and $\beta$ can be found using Equations (11)-(14). The coordinates of the

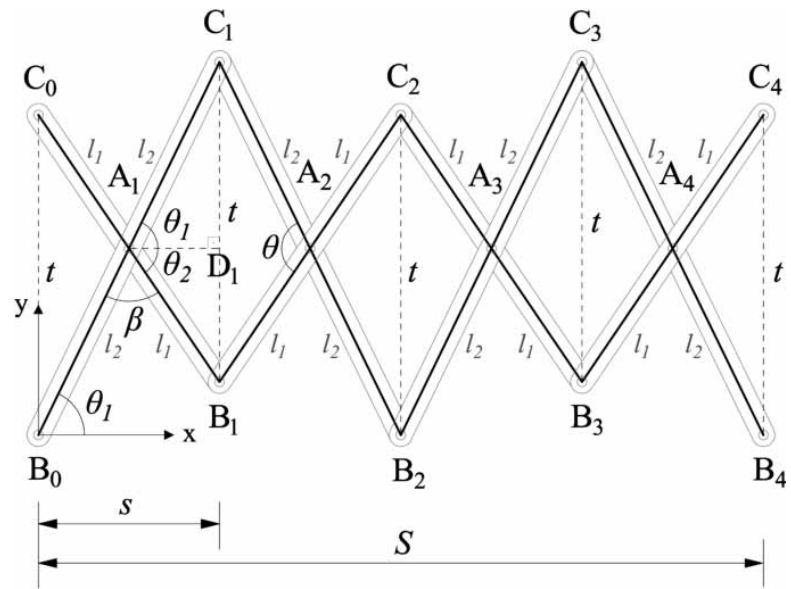

Figure 5| Rectilinear SSM with different bars and with scissor hinges at their midpoints 


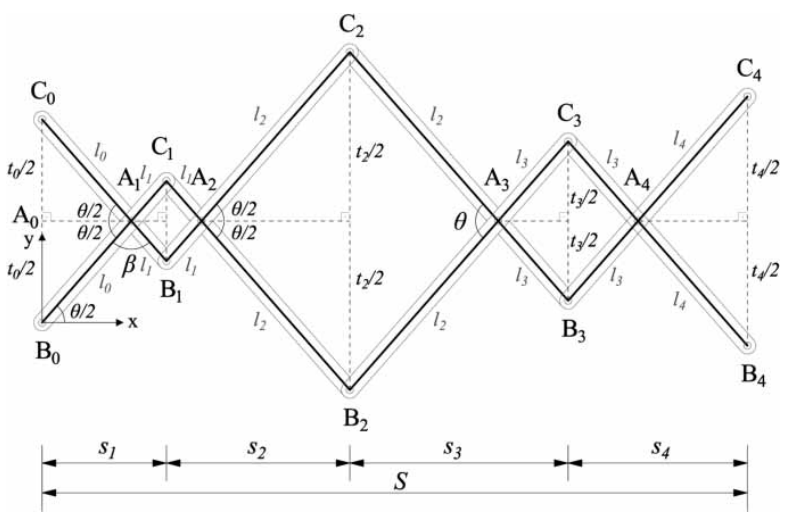

Figure 6 | Rectilinear SSM with arbitrary bar lengths and with scissor hinges eccentrically placed

$N$ th points of SUs are:

$$
\begin{gathered}
x_{B_{N}}=x_{C_{N}}=N\left(2 l_{2} \cos \theta_{1}\right)=N\left(2 l_{1} \cos \theta_{2}\right) \\
y_{B_{2 N}}=0 \text { and } y_{B_{2 N+1}}=\left(2 l_{2} \sin \theta_{1}\right)-t \\
y_{C_{2 N}}=t \text { and } y_{C_{2 N+1}}=2 l_{2} \sin \theta_{1}
\end{gathered}
$$

The fourth type of rectilinear SSM possesses arbitrary bar lengths and a scissor hinge eccentrically placed (Figure 6). In this type, each unit has different bar lengths and its scissor hinge is located randomly. Nevertheless, the unit lines are still parallel and translate without rotation. The condition for this type can be written as

$$
\begin{aligned}
a_{i-1} & =b_{i-1}=l_{0}, a_{i}=a_{i+1}=b_{i}=b_{i+1} \\
& =l_{1}, \ldots, a_{n}=a_{n+1}=b_{n}=b_{n+1}=l_{n}
\end{aligned}
$$

While $s$ and $t$ are same for each SU in the first and second type of rectilinear SSMs, it varies in arbitrary units, but angles $\theta$ and $\beta$ remain constant. Based on $\theta$ and $L$, the span and the unit thickness of $N$ th $\mathrm{SU}$ can be calculated as:

$$
\begin{aligned}
& s_{N}=\left(l_{n}+l_{n+1}\right) \cos \frac{\theta}{2} \\
& t_{N}=\sqrt{2 l_{n}^{2}(1-\cos \theta)}
\end{aligned}
$$

The $x$ coordinates of the $N$ th SU can be found according to origin $B_{0}$ of the system. However, it is hard to generalize a formula for the $y$ coordinates of the $N$ th unit, owing to the changing unit thicknesses. Therefore, it is calculated according to reference system $A_{0}$ :

$$
\begin{gathered}
x_{B_{N}}=x_{C_{N}}=\cos \frac{\theta}{2}\left[\left(l_{0}+l_{1}\right)+\left(l_{1}+l_{2}\right)\right. \\
\left.+\left(l_{2}+l_{3}\right)+\cdots+\left(l_{n}+l_{n+1}\right)\right] \\
y_{B_{N}}=y_{C_{N}}=\mp \frac{t_{n}}{2}
\end{gathered}
$$

The rectilinear SSMs examined above are commonly used for portative furniture elements such as foldable chairs or tables and for deployable structures such as tents and canopies. Because they are very simple mechanisms, they can easily be adapted to large-scale architectural applications.

\section{Polar units}

A polar unit is formed with units of straight bars by moving the scissor hinges away from the midpoints of the bars. By this means, scissor hinges of the system generate a curvature during deployment. The top and bottom hinges and the scissor hinges lie on concentric circles. The unit lines intersect at the centre of these circles at angle $\varphi$, which varies as the unit deploys. The intersection point moves closer to the unit as the curvature increases (You and Pellegrino, 1997).

Two types of curvilinear SSMs can be generated. The first type is with identical bars, with scissor hinges eccentrically placed (Figure 7). The deployability condition for this type is

$$
\frac{a_{i-1}}{b_{i-1}}=\frac{a_{i}}{b_{i}}=\frac{a_{i+1}}{b_{i+1}}=\cdots=\frac{a_{n}}{b_{n}}
$$

The bars are identical; therefore, it can be written that

$$
\begin{aligned}
& a_{i-1}=a_{i}=a_{i+1}=\cdots=a_{n}=l_{1} \\
& b_{i-1}=b_{i}=b_{i+1}=\cdots=b_{n}=l_{2}
\end{aligned}
$$

In designing a curvilinear SSM with fixed span, the span of the whole system $(S)$, the height $(h)$ and segment angle $(\varphi)$ should be defined to find the length of bars $(L)$, the number of SU $(N)$, unit thickness $(t)$, deployment angle $(\beta)$, radius of the base curve $\left(R_{b}\right)$ and SSM's angle $(\alpha)$. In this method, first, $R_{b}$ and $\alpha$ are calculated based on the $S$ and $h$. According to Pythagoras' theorem and the law of sinus for $\mathrm{MOB}_{5}$ triangle:

$$
\begin{gathered}
R_{b}=\frac{4 h^{2}+S^{2}}{8 h} \\
\alpha=2 \sin ^{-1}\left[\frac{S}{2 R_{b}}\right]
\end{gathered}
$$

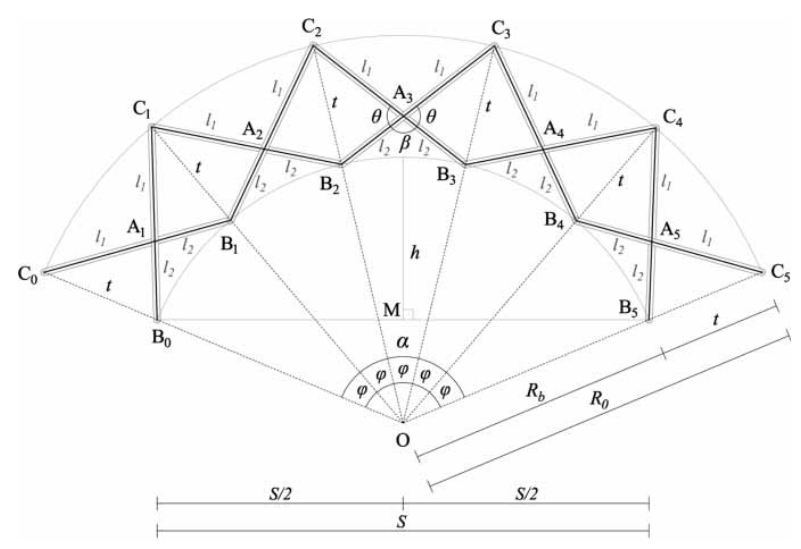

Figure $7 \mid$ Curvilinear SSM with identical bars and with scissor hinges eccentrically placed 
After finding $R_{b}$ and $\alpha$, the base curve is divided into angular portions with polar unit lines that intersect at centre $O$. The number of portions may be variable, but $\varphi$ must be the same for each segment so as to obtain a regular curvilinear system. According to determined $\varphi, N$ is found as

$$
N=\frac{\alpha}{\varphi}
$$

To find the length of the bars, the dimension of $t$ needs to be determined. According to chosen $t$, the radius of the outer curve $\left(R_{o}\right)$ and then the length of the bars can be calculated as

$$
R_{0}=R_{b}+t
$$

Polar units have identical bars $(L)$, but are divided into two unequal semi-bars, $l_{1}$ and $l_{2}$. Therefore, the length of the bar is equal to

$$
L=l_{1}+l_{2}
$$

$L$ can be found using the cosine rule to the $B_{2} \mathrm{OC}_{3}$ triangle in Figure 8:

$$
L^{2}=R_{b}^{2}+R_{0}^{2}-2 R_{b} R_{0} \cos \varphi
$$

It is possible to write Equations (35) and (36) through the similarity of triangles:

$$
\frac{R_{b}}{R_{0}}=\frac{m}{n}
$$

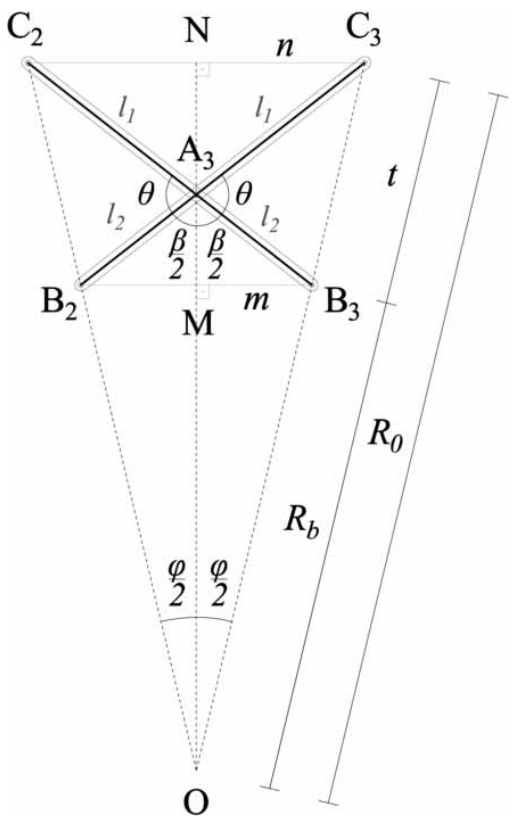

Figure $8 \mid$ A segment of curvilinear SSM in Figure 7

$$
\frac{l_{2}}{l_{1}}=\frac{m}{n}
$$

Equating (35) and (36),

$$
\frac{R_{b}}{R_{0}}=\frac{l_{2}}{l_{1}} \Rightarrow l_{1}=\frac{R_{0}}{R_{b}} l_{2} \quad \text { and } \quad l_{2}=\frac{R_{b}}{R_{0}} l_{1}
$$

Substituting Equation (33) into (37) results in

$$
l_{1}=\frac{L R_{0}}{R_{b}+R_{0}} \quad \text { and } \quad l_{2}=\frac{L R_{b}}{R_{b}+R_{0}}
$$

To find $\beta$, the parameter of $m$ should be calculated:

$$
\begin{aligned}
m & =l_{2} \sin \frac{\beta}{2} \\
m & =R_{b} \sin \frac{\varphi}{2}
\end{aligned}
$$

By equating (39) and (40), $\beta$ is obtained as

$$
\beta=2 \sin ^{-1}\left[\frac{R_{b}}{l_{2}} \sin \frac{\varphi}{2}\right]
$$

To understand its deployment behaviour and to show its geometric transformation capacity, a model of curvilinear SSM, which consists of SUs whose bars are identical and scissor hinges are eccentrically placed, was constructed (Figure 9). Such SSMs may offer viable solutions for architectural applications due to their deployment behaviour that provides great advantages of speed and of ease of erection and dismantling in comparison to conventional ones, and it is easier to expand or remove the structures when necessary. Furthermore, they become self-standing structures by adding an extra link to the system; therefore, large supports on the ground are not required. This property increases the feasibility for this kind of SSM to be used as tents or portative shed covering.

The second type of curvilinear SSM is with arbitrary units (Figure 10). These units have different bar lengths, and their scissor hinges are not located at the midpoints of the bars. Its condition is

$$
\frac{a_{i-1}}{b_{i-1}} \neq \frac{a_{i}}{b_{i}} \neq \frac{a_{i+1}}{b_{i+1}} \neq \cdots \neq \frac{a_{n}}{b_{n}}
$$

In this type, lengths of bars can vary. However, the lengths on both sides of the unit lines should be equal to each other so as stay in accordance with the general deployability conditions:

$$
\begin{gathered}
a_{i}=a_{i+1}, b_{i}=b_{i+1}, a_{i+2}=a_{i+3}, \\
b_{i+2}=b_{i+3}, \ldots, a_{n}=a_{n+1}, b_{n}=b_{n+1}
\end{gathered}
$$

Suppose that the parameters of $S$ and $h$ are the same as the first type of polar unit above. $R_{b}$ and $\alpha$ can be found according to $S$ and $h$. However, the base curve should be divided into unequal angular portions to obtain arbitrary SUs. This 

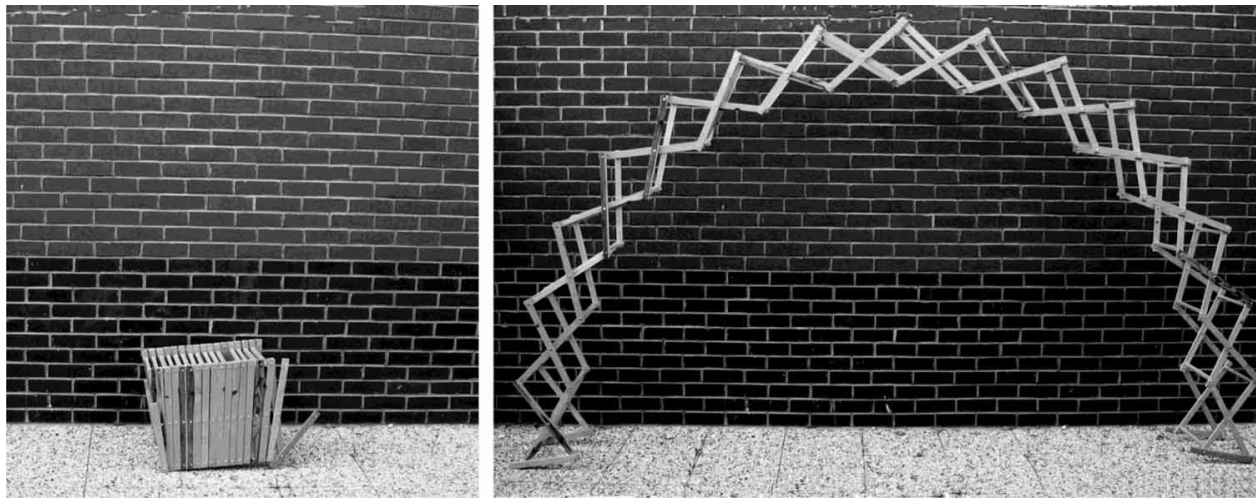

Figure 9|Model of curvilinear SSM with identical bars and with scissor hinges eccentrically placed

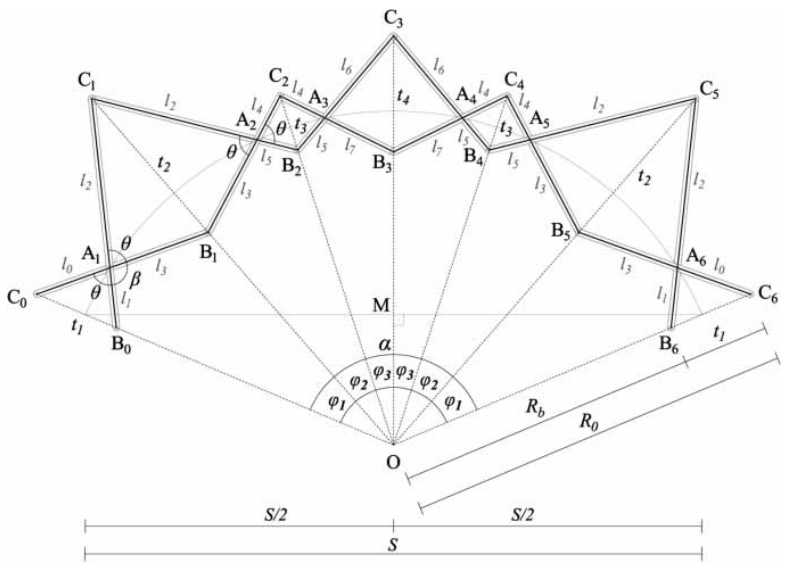

Figure 10|Curvilinear SSM with arbitrary units

means that each SU will have different $L, t$ and $\varphi$, but that $\theta$ and $\beta$ will remain the same. Therefore, it is difficult to generalize solutions and rules for arbitrary systems. It requires the use of matrices because of the numbers of unknown parameters.

\section{Angulated units}

Discovered by Chuck Hoberman, therefore known as Hoberman units, angulated units consist of two identical angulated bars rather than straight ones. Each angulated bar has a central kink of amplitude $\omega$. The segment angle $(\varphi)$ between two angulated bars is constant during deployment, whereas it varies in polar units (Jensen, 2004). While the kink angle $\omega$ is initially independent of the segment angle $\varphi$, as shown in Figure 11, in extension the relationship between $\varphi$ and $\psi$ can be written as

$$
\psi=\frac{\varphi}{2}
$$

Equation (44) shows that angulated units can be used for radially deploying closed-loop structures. While

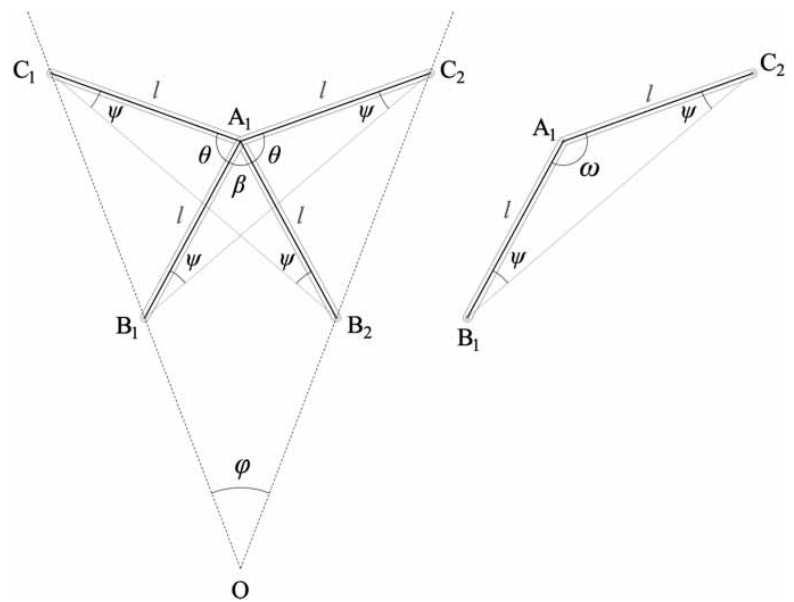

Figure 11 | Angulated unit

translational and polar units show rectilinear and curvilinear deployments, angulated units are capable of retracting to their own perimeters.

Two different types of SSMs can be derived, according to the assembly method. In the first type, unit lines intersect at centre $O$. To generate a radially deployable closed ring structure, angulated units should be connected at their end nodes of $B_{N}$ and $C_{N}$. Using the geometry of polar units with constant bar lengths and scissor hinges in Figure 7, the geometry of angulated units can be derived (Figure 12). Therefore, unknown parameters of angulated units can be easily calculated according to those parameters that are shown in Figure 7. The condition for this angulated unit is

$$
\begin{aligned}
a_{i-1} & =b_{i-1}=a_{i}=b_{i}=a_{i+1} \\
& =b_{i+1}=\cdots=a_{n}=b_{n}=l
\end{aligned}
$$

For the same length of span $(S)$ and height $(h)$ as described above in Figure 7, the radius of the base curve $\left(R_{b}\right)$, the radius of the outer curve $\left(R_{o}\right)$, the length of bars $(L)$, 


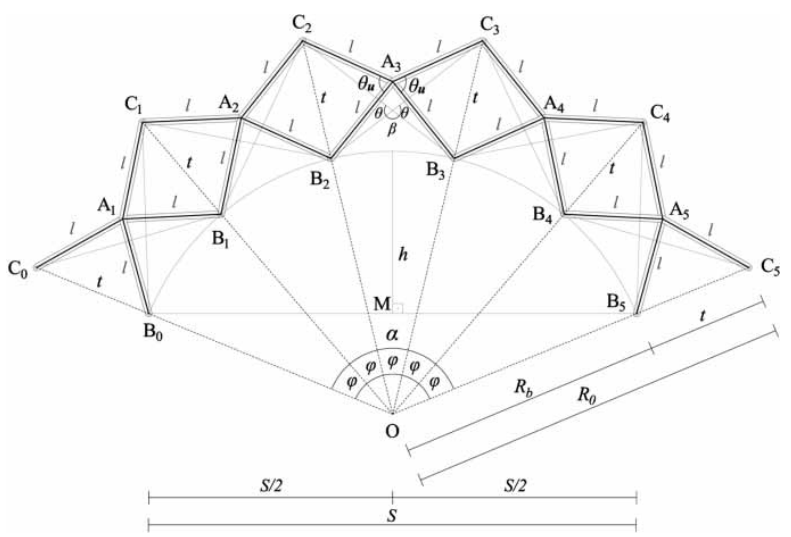

Figure $12 \mid$ Partial radially deployable closed ring SSM with angulated units

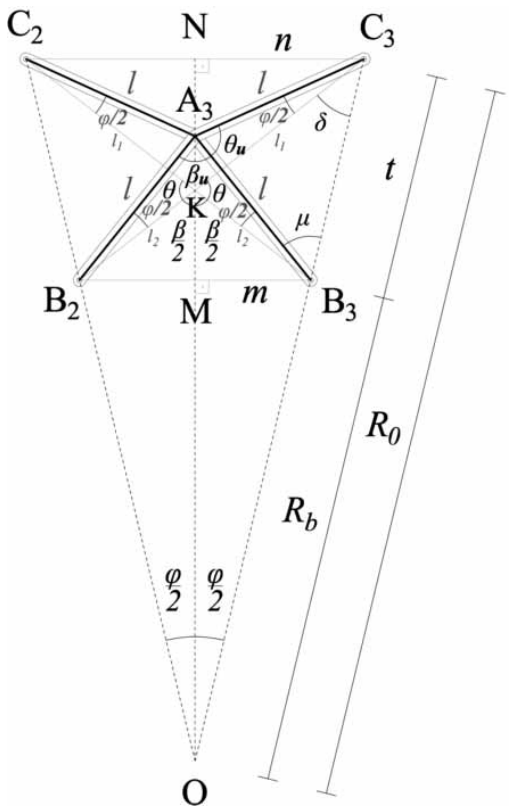

Figure 13 |A segment of radially deployable closed ring SSM in Figure 12

SSM's angle $(\alpha)$, deployment angle $(\beta)$ and segment angle $(\varphi)$ of angulated units can be found. According to $A_{3} B_{2} K$ triangle in Figure 13,

$$
\frac{\varphi}{2}+\frac{\beta_{u}}{2}=\frac{\beta}{2} \Rightarrow \beta_{u}=\beta-\varphi
$$

The law of sinus for $C_{3} K B_{3}$ triangle gives

$$
\frac{t}{\sin \theta}=\frac{l_{2}}{\sin \delta} \Rightarrow \delta=\sin ^{-1}\left[\frac{l_{2}}{t} \sin \theta\right]
$$

For $C_{3} A_{3} B_{3}$ triangle, it can be written that

$$
\begin{gathered}
\mu=\frac{\varphi}{2}+\delta \\
\theta_{u}=\pi-2 \mu
\end{gathered}
$$

Using sinus in the $C_{3} A_{3} B_{3}$ triangle, the length of the bar can be calculated as

$$
\frac{t}{\sin \theta_{u}}=\frac{l}{\sin \mu} \Rightarrow l=t \frac{\sin \mu}{\sin \theta_{u}}
$$

Considering the geometric principles of angulated units, an architectural model was built (Figure 14). To stabilize the system, two partial ring SSMs were connected to each other. By parallel multiplication of each system, such SSMs may be used for architectural applications such as temporary buildings, emergency shelters, exhibition halls, sport venues or for military applications.

In the second type of angulated units, unit lines are parallel to each other and remain so during the deployment. To keep the unit lines parallel, angulated units should be connected at their end nodes of $B_{N}$ and $B_{N+1}$. By this means, the SSM translates. As shown in Figure 15, the entire system is a segment of a circle whose centre lies at point $O$ and whose segment angle is $\varphi$. The condition for this type is

$$
\begin{aligned}
a_{i-1} & =b_{i-1}=a_{i}=b_{i}=a_{i+1} \\
& =b_{i+2}=\cdots=a_{n}=b_{n}=l
\end{aligned}
$$

To solve the system, at least three parameters should be given. The known parameters are $\varphi, R_{0}$ and the number of SUs in one segment $\left(N_{s}\right)$. According to Equation (44), the bar geometry has to be such that $\psi=\varphi / 2$. Therefore, the kink angle is

$$
\omega=180-2 \psi
$$

The unknown parameters of $R_{b 1}, \beta_{u 1}, \theta_{u 1}, R_{b 2}, \beta_{u 2}, \theta_{u 2}$, $R_{b 3}$ and $\beta_{u 3}$ can be calculated as follows:

$$
\begin{gathered}
R_{b 1}=\sqrt{2 l^{2}(1-\cos \omega)} \\
\beta_{u 1}=360-2 \omega \\
\theta_{u 1}=\omega-\beta_{u 1} \\
R_{b 2}=\sqrt{2 l^{2}\left(1-\cos \theta_{u 1}\right)} \\
\beta_{u 2}=360-\left(2 \theta_{u 1}+\beta_{u 1}\right) \\
\theta_{u 2}=\omega-\beta_{u 2} \\
R_{b 3}=\sqrt{2 l^{2}\left(1-\cos \theta_{u 2}\right)} \\
\beta_{u 3}=360-\left(2 \theta_{u 2}+\beta_{u 2}\right)
\end{gathered}
$$

The sum of $R_{1}, R_{2}$ and $R_{3}$ is equal to

$$
R_{0}=R_{b 1}+R_{b 2}+R_{b 3}
$$

By solving Equation (61), $l$ can be found. 

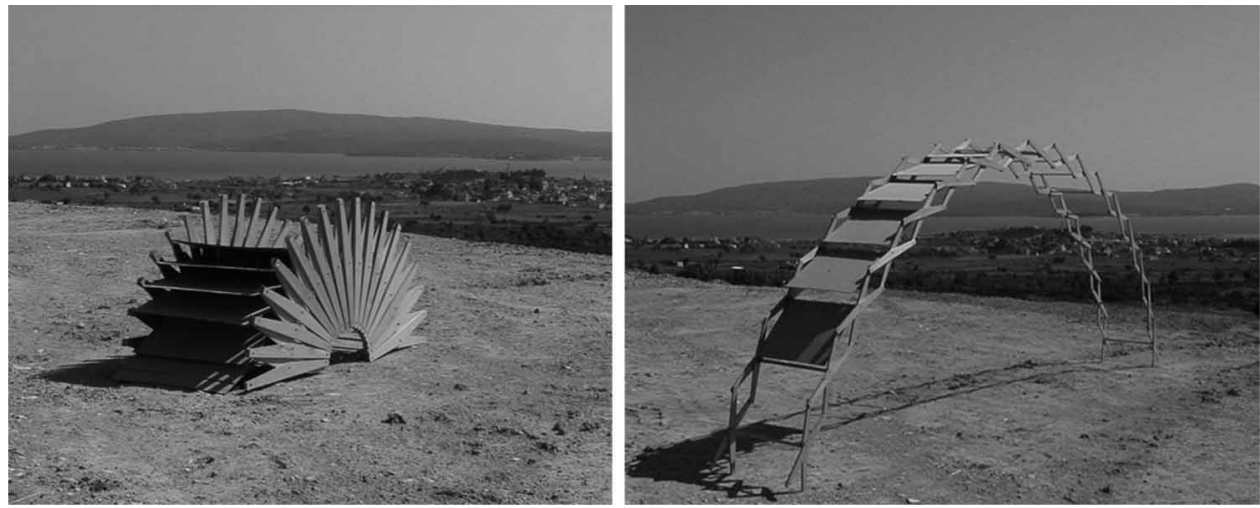

Figure 14 | Model of partial radially deployable closed ring SSM with angulated units

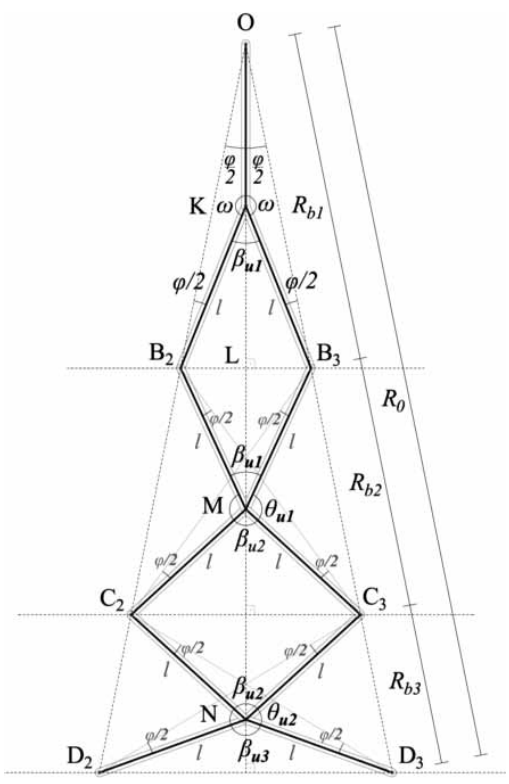

Figure 15|Rectilinear SSM with angulated units

\section{ASSEMBLIES OF MIXED UNITS}

This section aims to construct planar SSMs with mixed SUs with respect to the general deployability condition. Using three types of SUs in different combinations, it is possible to construct new SSMs in different forms. Such planar SSMs lead to the design of 3D scissor structures for further architectural applications. In the light of this assumption, a few planar models were constructed.

The following examples are generated by determining the position of scissor hinges or of bar lengths based on the general deployability condition. In the first example, bars are identical and the location of pivot points is constant, but scissor hinges are not located at the midpoints of the bars (Figure 16).
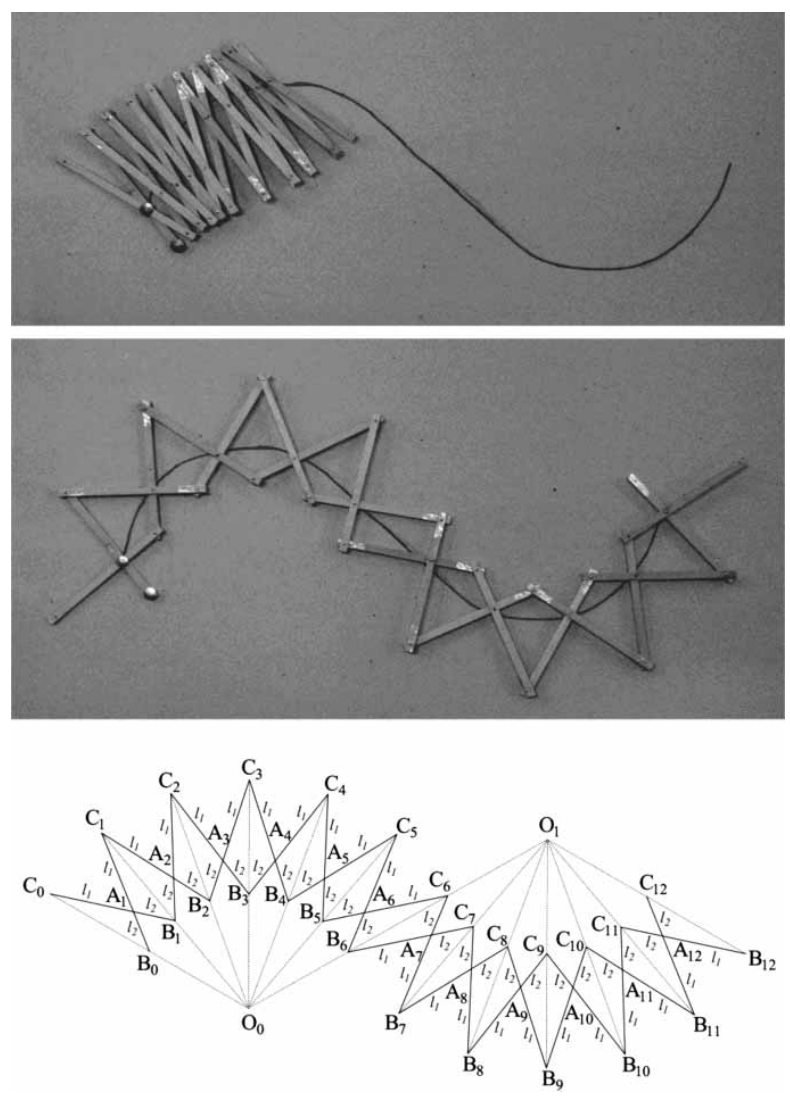

Figure 16|Curvilinear SSM with identical polar units

To construct such mixed SSM, first, an SSM with $O_{1}$ centre is designed. Then, by reversing the first SSM, a second SSM with $\mathrm{O}_{2}$ centre is generated. These two SSMs are connected to each other at points $B_{6}$ and $C_{6}$. As in Escrig's equation, the sum of the lengths of the bars is equal on both sides of the unit line that passes through $O_{0}$ and $O_{1}$. Therefore, $l_{1}+l_{2}=l_{2}+l_{1}$. 

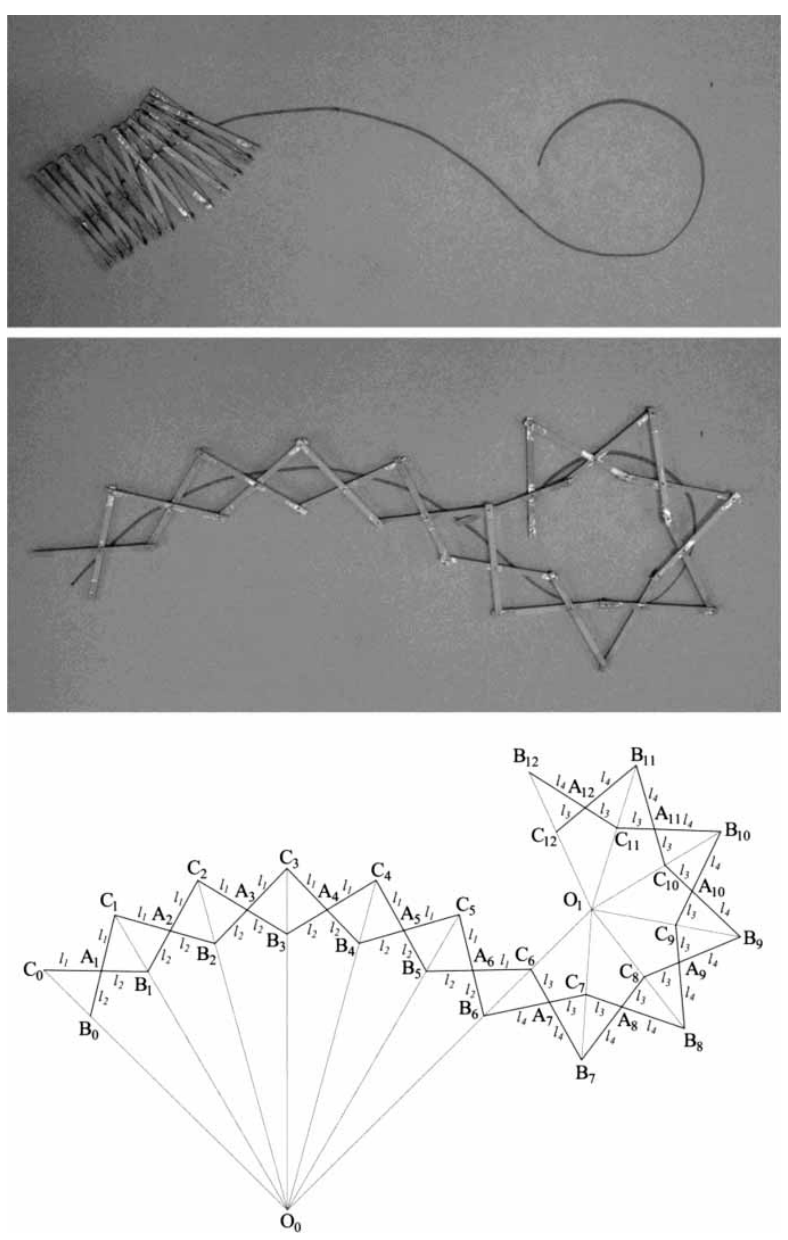

Figure 17|Curvilinear SSM with two different polar units

A second example of mixed units is composed of two SSMs whose curvatures are different (Figure 17). Both SSMs have the same bar length $L$, but the location of scissor hinges is different. The bar length of SSM whose centre is $O_{0}$ is $L=l_{1}+l_{2}$ whereas it is $L=l_{3}+l_{4}$ in the second SSM whose centre is $O_{1}$. The curvatures of these SSMs depend on the lengths of $l_{2}$ and $l_{3}$. In this example, $l_{3}<l_{2}$. To comply with the general deployability condition, the sum of the lengths of the bars on both sides of the unit line that passes through $O_{0}$ and $O_{1}$ must equal to each other. Therefore, $l_{1}+l_{2}=l_{3}+l_{4}$.

The SSMs in Figures 16 and 17 can be used as deployable roof structures or portative walls that can offer different curvilinear geometries.

The third mixed SSM is composed of one translational part and two polar parts (Figure 18). The bar lengths are $L_{2}=2 l_{2}+l_{4}, L_{2}=2 l_{2}+l_{4}, L_{3}=l_{2}+l_{3}$ and $L_{4}=l_{3}+l_{4}$. Up to points $A_{3}$ and $A_{4}$, the SSM translates because unit lines 1,2 and 3 are parallel to each other and remain parallel during the deployment. The sums of the lengths of the bars
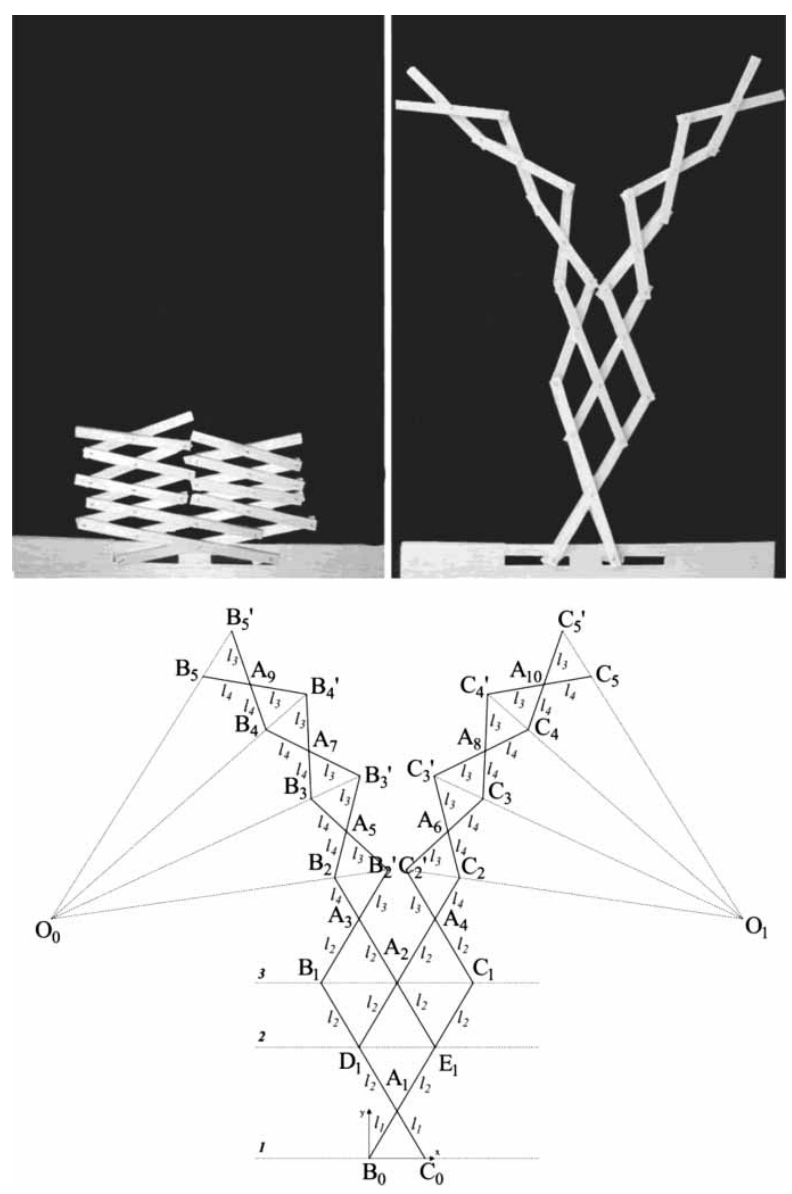

Figure 18 | Curvilinear SSM with translational and polar units

are equal on both sides of unit lines 2 and 3. Therefore, $l_{2}+l_{2}=l_{2}+l_{2}$. The translational part and the polar part whose centre is $O_{0}$ are connected to each other at points $B_{2}$ and $B_{2}{ }^{\prime}$. The sum of the lengths of the bars is equal on both sides of the unit line that passes through $O_{0}$ and $B_{2}$. Thus, $l_{3}+l_{4}=l_{3}+l_{4}$.

As can be understood from SSMs in Figures 16-18, various geometries can be obtained by the attachment of different SUs. By using these combinations, not only architectural elements and building components but also various furnitures and tools can be developed.

\section{CONCLUSION}

This article has systematically analysed the geometric principles and design methods of planar SSMs with respect to their basic typologies, design rules and constraints. It has been demonstrated that deployment behaviours of SSMs are related to the basic unit types of translational, polar and angulated units, which are used 
to assemble the entire structure. Each unit type has been examined in detail. Conditions to obtain different types of rectilinear and curvilinear SSMs have been discussed.
According to the given parameters of SSMs, unknown parameters of the system have been calculated using basic trigonometry.

\section{References}

Akgün, Y., Gantes, C.J., Kalochairetis, K.E. and Kiper, G., 2010a, 'A novel concept of convertible roofs with high transformability consisting of planar scissor-hinge structures', Engineering Structures 32(9), 2873-2883.

Akgün, Y., Korkmaz, K., Sobek, W. and Gantes, C.J., 2010b, 'A novel transformable roof system with spatial scissor-hinge structural mechanisms', in Y. Akgün and E. Gezgin (eds), Symposium Proceedings of AZcIFToMM 2010, International Symposium of Mechanism and Machine Science, Izmir, Izmir Institute of Technology Press, 116-123.

Al Khayer, M. and Lalvani, H., 1998, 'Scissors-action deployables based on space-filling of polygonal hyperboloids', in S. Pellegrino and S. Guest (eds), Proceedings of IUTAM_IASS Symposium on Deployable Structures: Theory and Applications, Cambridge, Kluwer Academic Publishers, 1-10.

Atake, K., 2000, New variations of the scissors technique, MARAS III: International Conference on Mobile and Rapidly Assembled Structures, Madrid, WIT Press, 143-154.

Block, P., 2003, 'Scissor hinge deployable membrane structures tensioned by pleated pneumatic artificial muscles', Master thesis, Vrije Universiteit Brusel.

Buhl, T., Jensen, F.V. and Pellegrino, S., 2004, 'Shape optimization of cover plates for retractable roof structures', Computer and Structures 82, 1227-1236.

Chen, W., Fu, G., Gong, J., He, Y. and Dong, S., 2002, 'A new design conception for large span deployable flat grid structures', International Journal of Space Structures 17(4), 293-299.

Clarke, R., 1984, 'The kinematics of a novel deployable space structure system', in H. Nooshin (ed), Proceedings of the 3rd International Conference on Space Structures, London, Elsevier Science Publishers, 820-822.

De Temmerman, N., 2007, 'Design and analysis of deployable bar structures for mobile architectural applications', PhD dissertation, Vrije Universiteit Brussel.

Dieguez, R. and Cozar, J., 1998, 'Florin system double layer spatial deployable structures, with frames of rhombuses and scissors', in S. Pellegrino and S. Guest (eds), Proceedings of
IUTAM_IASS Symposium on Deployable Structures: Theory and Applications, Cambridge, Kluwer Academic Publishers, 117-126.

Escrig, F., 1984, 'Expandable space frame structures', in $\mathrm{H}$. Nooshin (ed), Proceedings of the 3rd International Conference on Space Structures. London, Elsevier Science Publishers, 845-850.

Escrig, F., 1985, 'Expendable space structures', International Journal of Space Structures 1(2), 79-91.

Escrig, F. and Valcarcel, J., 1986a, 'Analysis of expandable space bar structures', in K. Heki (ed), Proceedings of IASS Symposium on Shells, Membranes, and Space Frames, vol. 3, Osaka, Elsevier Science Publishers, 269-276.

Escrig, F. and Valcarcel, J., 1986b, 'Great size umbrellas with expendable bar structures', Proceedings of the 1st International Conference on Lightweight Structures in Architecture, Sydney, University of New South Gales, 676-681.

Escrig, F. and Valcarcel, J., 1987, 'Curved expandable space grids', Proceedings of the International Conference on the Design and Construction of Non-Conventional Structures, Edinburg, Civil Comp Press, 157-168.

Escrig, F. and Valcarcel, J., 1993, 'Geometry of expandable space frame structures', International Journal of Space Structures 8(1-2), 71-84.

Gan, W. and Pellegrino, S., 2003, 'Closed-loop deployable structures', Proceedings of 44th AIAA/ASME/ ASCE/AHS/ASC Structures, Structural Dynamics and Materials Conference, Norfolk, VA AIAA 2003-1450.

Gan, W. and Pellegrino, S., 2006, 'Numerical approach to the kinematic analysis of deployable structures forming a closed loop', Journal of Mechanical Engineering Science 220(7), 1045-1056.

Gantes, C., 2001, Deployable Structures: Analysis and Design, Boston, WIT Press.

Gantes, C. and Konitopoulou, E., 2004, 'Geometric design of arbitrarily curved bi-stable deployable arches with discrete joint size', International Journal of Solids and Structures 41(20), 5517-2240.

Gantes, C., Logcher, R., Connor, J. and Rosenfeld, Y., 1993, 'Deployability conditions for curved and flat, polygonal and trapezoidal deployable structures', International Journal of Space Structures 8(1\&2), 97-106.

Gantes, C., Connor, J. and Logcher, R., 1994, 'A systematic design methodology for deployable structures', International Journal of Space Structures 9(2), 67-86.

Gutierrez, E. and Valcarcel, J., 2002, 'Generation of foldable domes formed by bundle modules with quadrangular base', Journal of the International Association for Shells and Spatial Structures 43(140), 133-140.

Hoberman, C., 1990. 'Reversibly expandable doubly-curved truss structure', United States Patent No. 4, 942, 700.

Jensen, F., 2004, 'Concepts for retractable roof structures', PhD dissertation, University of Cambridge.

Kaveh, A. and Davaran, A., 1996, 'Analysis of pantograph foldable structures', Computers \& Structures 59(1), 131-140.

Kokawa, T. and Hokkaido, T., 1997, 'Cable scissors arch-marionettic structure: structural morphology, towards the new millennium', Proceedings of International Conference of IASS, Nottingham, University of Nottingham, 107-114.

Kwan, A., 1995, 'A parabolic pantographic deployable antenna', International Journal of Space Structures 10(4), 195-203.

Kwan, A., You, Z. and Pellegrino, S., 1993, 'Active and passive cable elements in deployable retractable masts', International Journal of Space Structures 8(1\&2), 29-40.

Langbecker, T., 1999, 'Kinematic analysis of deployable scissor structures', International Journal of Space Structures 14(1), 1-15.

Nagaraj, B.P., Pandiyan, R. and Ghosal, A., 2009, 'Kinematics of pantograph masts', Mechanism and Machine Theory 44, 822-834.

Patel, J. and Ananthasuresh, G.K., 2007, 'A kinematic theory for radially foldable planar linkages', International Journal of Solids and Structures 44, 6279-6298.

Pinero, E., 1961, 'Project for a mobile theatre', Architectural Design 12, 570.

Rosenfeld, Y. and Logcher, R.D., 1988, 'New concepts for deployable collapsable structures', International Journal of Space Structures 3(1), 20-32. 
Van Mele, T., De Temmerman, N., De Laet, L. and Mollaert, M., 2007, 'Retractable roofs: scissor-hinged membrane structures', Proceedings of the TensiNet Symposium 2007 -

Ephemeral Architecture: Time and Textiles, Milan, Liberia CLUP.
You, Z. and Pellegrino, S., 1997, 'Foldable bar structures', International Journal of Solids and Structures 34(15), 18251847.

Zanardo, A., 1986, 'Two dimensional articulated systems deployable on a single or double curvature structure', Meccanica 21, 106-111.

Zhao, J. and Feng, Z., 2009,

'The mechanism theory and application of deployable structures', Mechanism and Machine Theory 44, 324-335. 